\title{
On concentrated probabilities
}

\author{
by Wojciech Bartoszek (Pretoria)
}

\begin{abstract}
Let $G$ be a locally compact Polish group with an invariant metric. We provide sufficient and necessary conditions for the existence of a compact set $A \subseteq G$ and a sequence $g_{n} \in G$ such that $\mu^{* n}\left(g_{n} A\right) \equiv 1$ for all $n$. It is noticed that such measures $\mu$ form a meager subset of all probabilities on $G$ in the weak measure topology. If for some $k$ the convolution power $\mu^{* k}$ has nontrivial absolutely continuous component then a similar characterization is obtained for any locally compact, $\sigma$-compact, unimodular, Hausdorff topological group $G$.
\end{abstract}

1. Introduction. Let $G$ be a locally compact Hausdorff group. For a probability (Radon) measure $\mu$ on $G$ the question under what conditions on $\mu$,

$$
\lim _{n \rightarrow \infty} \sup _{g \in G} \mu^{* n}(g A)=0 \quad(A \text { compact })
$$

holds was raised by K. H. Hofmann and A. Mukherjea in 1981 (see [HM]). If $G$ is Abelian the situation is clear since 1984 when Y. Derriennic and M. Lin (see [DL1]) proved the following:

If $G$ is a commutative, locally compact, noncompact topological group, then $(*)$ holds if and only if there is no compact subgroup $H \subseteq G$ such that for some $g \in G$ we have $\mu(g H)=1$.

In 1991 similar conditions were found for all countable groups (see [B2], [B3]). For "continuous" noncommutative groups such conditions are not provided yet. In [DL2] only sufficient conditions for $(*)$ are shown.

The aim of this paper is to extend some results of [B2], [B3], [DL1] and [DL2] to a class of groups wider than Abelian or discrete groups. A suggestion it is possible appears for instance in [B1] where it is pointed out that whenever left and right uniform structures on a noncompact group $G$ are equivalent, then $(*)$ holds for any adapted and strictly aperiodic probability measure $\mu$ on $G$ (for definitions, see below). Clearly left and right uniform

1991 Mathematics Subject Classification: 22D40, 43A35, 47A35, 60B15, 60J15.

Key words and phrases: random walk, concentration function, convolution operator. 
structures are equivalent for any Abelian or discrete group $G$. In Sections 3 and 4 we will consider metrizable locally compact groups. For that class we fully describe the set of measures satisfying $(*)$ as well as determine its Baire category.

In the last Section 5 we discuss the convergence $(*)$ with the additional assumption that $\mu$ is spread out. In this case it is sufficient to consider unimodular groups. We establish necessary and sufficient conditions for this convergence in terms of properties of the support of $\mu$.

2. Preliminaries. In this part of the paper we recall definitions, introduce notations, and quote necessary results concerning asymptotic behaviour of iterates of convolution operators. Most of them can be found in [DL2], but for the convenience of the reader they are collected here.

$G$ always denotes a topological Hausdorff group which is at least locally compact and $\sigma$-compact. By $e$ we denote its neutral element. The Abelian case is easy and known, thus our study is of interest for noncommutative groups. We may always assume that the group $G$ is noncompact (obviously on a compact group each probability measure is concentrated). If $G$ is metrizable, the metric is denoted by $d$. We fix a right Haar measure $\lambda$ on the $\sigma$-algebra $\mathcal{B}$ of all Borel subsets of $G$. All measures considered in this paper are Borel and regular (Radon). The (AL) Banach lattice of all bounded real signed measures on $(G, \mathcal{B})$ is denoted by $M(G)$. The convex subset of all probability measures on $G$ is denoted by $P(G)$. If $\nu_{1}, \nu_{2} \in M(G)$ then $\nu_{1} * \nu_{2}$ stands for their convolution. The support $S(\mu)$ of a measure $\mu \in P(G)$ is the smallest closed set $F$ such that $\mu(F)=1$. It is well known that if $\nu_{1}, \nu_{2} \in P(G)$ then $\nu_{1} * \nu_{2} \in P(G)$ and $S\left(\nu_{1} * \nu_{2}\right)=\overline{S\left(\nu_{1}\right) S\left(\nu_{2}\right)}$. We denote by $\check{\mu}$ the symmetric reflection of a measure $\mu$ (i.e. $\check{\mu}(A)=\mu\left(A^{-1}\right)$ for all $A \in \mathcal{B})$. Clearly $S(\check{\mu})=S(\mu)^{-1}$.

There are two topologies on $P(G)$ important for us. The first one is the so-called weak measure topology, and is introduced by its base sets:

$$
\begin{aligned}
\mathcal{U}\left(\mu, f_{1}, \ldots, f_{k}, \varepsilon_{1}, \ldots, \varepsilon_{k}\right) & \\
= & \left\{\nu \in P(G):\left|\int_{G} f_{j} d \nu-\int_{G} f_{j} d \mu\right|<\varepsilon_{j}, j=1, \ldots, k\right\}
\end{aligned}
$$

where $f_{1}, \ldots, f_{k}$ are bounded continuous functions and $\varepsilon_{1}, \ldots, \varepsilon_{k}$ are positive numbers. It is well known that $P(G)$ is a Polish space for this topology if and only if $G$ is a Polish group. The second topology is the so-called vague topology and its base sets are described by (1) but with the restriction that the functions $f_{1}, \ldots, f_{k}$ are compactly supported. Similarly we introduce these two topologies in the closed, convex set $M^{\leq 1}(G)=\{\nu: \nu \geq$ 0 and $\nu(G) \leq 1\}$. Let us recall that $M^{\leq 1}(G)$ with the vague topology is a 
compact Hausdorff space and $(P(G), *)$ with the weak measure topology is a topological semigroup (see $[\mathrm{H}]$, Theorem 1.2.2). More details concerning these topologies can be found in $[\mathrm{H}]$ and $[\mathrm{P}]$.

The average operator $P_{\mu}$ associated with a given measure $\mu \in M(G)$ is defined as the convolution operator $P_{\mu} f(g)=\int_{G} f(g t) d \mu(t)$. If $\mu \in P(G)$ then this operator is a positive linear contraction on the Banach lattice $C_{0}(G)$ of all continuous functions vanishing at infinity with the supremum norm $\|\cdot\|_{\infty}$ as well as on all $\left(L^{p}(\lambda),\|\cdot\|_{p}\right)$ spaces. The latter easily follows from the fact that the mapping $L^{p}(\lambda) \ni f(\cdot) \rightarrow f(\cdot t)=f_{t}(\cdot) \in L^{p}(\lambda)$ is an invertible linear isometry for any fixed $t \in G$ (by right invariance of $\lambda$ ). We also notice that for $p=2$ the adjoint operator to $P_{\mu}$ is exactly $P_{\breve{\mu}}$. By standard computations, the $n$th iterate of $P_{\mu}$ is the average operator $P_{\mu * n}$ corresponding to the $n$th fold convolution of $\mu$. The family of all convolution operators $\left\{P_{\mu}: \mu \in P(G)\right\} \subset \mathcal{L}\left(L^{p}(\lambda)\right)$ is denoted by $\mathcal{M}_{p}(G)$. It is well known that $\mathcal{M}_{2}(G)$, with the strong operator and weak operator topologies inherited from $\mathcal{L}\left(L^{2}(\lambda)\right)$, is homeomorphic to $P(G)$ with the weak measure topology (or the vague topology). The set $\mathcal{M}_{2}^{\leq 1}(G)=\left\{P_{\mu}: \mu \in M^{\leq 1}(G)\right\}$ equipped with the weak operator topology (inherited from $\mathcal{L}\left(L^{2}(\lambda)\right)$ ) and $M^{\leq 1}(G)$ with the vague topology are homeomorphic. The proofs of these two facts can be found in [H] (Lemma 6.1.23, p. 422).

For a while let us assume that the topology of $G$ is metrizable by some metric $d$. While a left (or right) invariant metric does always exist it is not necessarily so for an invariant metric. We say that a metric $d$ on $G$ is invariant if for any $g, g_{1}, g_{2} \in G$ we have

$$
d\left(g_{1}, g_{2}\right)=d\left(g g_{1}, g g_{2}\right)=d\left(g_{1} g, g_{2} g\right) .
$$

It is well known (see $[\mathrm{HR}]$, p. 109) that if $(G, d)$ is a metric group then there exists an invariant metric if and only if for any $x_{n} \in G$ such that $\lim _{n \rightarrow \infty} d\left(x_{n}, e\right)=0$ we have $\lim _{n \rightarrow \infty} \sup _{g \in G} d\left(g x_{n} g^{-1}, e\right)=0$ (the left and right uniform structures are equivalent).

A set $B \subseteq G$ is called $\varepsilon$-dense in $A \subseteq G$ if for every $g \in A$ there exists $y \in B$ such that $d(g, y) \leq \varepsilon$. For a fixed set $A \subseteq G$ the smallest $m$ such that there exists a finite set $\left\{y_{1}, \ldots, y_{m}\right\} \subseteq A$ which is $\varepsilon$-dense in $A$ is denoted by $N(A, \varepsilon)$. If no such $m$ exists we write $N(A, \varepsilon)=\infty$. The largest $l$ such that there exists a finite set $\left\{y_{1}, \ldots, y_{l}\right\} \subseteq A$ with $d\left(y_{j}, y_{i}\right) \geq \varepsilon, j \neq i$, is denoted by $L(A, \varepsilon)$. Clearly $N(A, \varepsilon) \leq L(A, \varepsilon)$. Obviously $N(A, \varepsilon)=N(\varphi(A), \varepsilon)$ and $L(A, \varepsilon)=L(\varphi(A), \varepsilon)$ for every invertible isometry $\varphi$ on $(G, d)$. By $K\left(g_{0}, r\right)$ where $r \geq 0$ we denote the open ball $\left\{g \in G: d\left(g_{0}, g\right)<r\right\}$, and $\bar{K}\left(g_{0}, r\right)$ stands for the closed ball $\left\{g \in G: d\left(g_{0}, g\right) \leq r\right\}$.

The distance from a point $g \in G$ to a nonempty set $A \subseteq G$ is denoted by $d(g, A)$ while $\operatorname{dist}(A, B)$ stands for the Hausdorff distance. If $A \subseteq G$ then $\overline{\mathbf{K}}(A, r)$ denotes the generalized closed ball $\{g \in G: d(g, A) \leq r\}$. 
We notice that if the metric $d$ is invariant then for all $A_{1}, A_{2} \subseteq G$ and $\varepsilon>0$ we have

$$
A_{1} \overline{\mathbf{K}}\left(A_{2}, \varepsilon\right) \subseteq \overline{\mathbf{K}}\left(A_{1} A_{2}, \varepsilon\right) \quad \text { and } \quad \overline{\mathbf{K}}\left(A_{2}, \varepsilon\right) A_{1} \subseteq \overline{\mathbf{K}}\left(A_{2} A_{1}, \varepsilon\right) .
$$

DEFINITION 1. The concentration function of a probability measure $\mu$ on $G$ is the set function $\mathrm{k}_{\mu}$ defined as

$$
\mathrm{k}_{\mu}(A)=\sup _{g \in G} \mu(g A) \quad \text { where } A \in \mathcal{B} \text {. }
$$

Definition 2. A probability measure $\mu$ on $G$ such that

$$
\lim _{n \rightarrow \infty} k_{\mu^{* n}}(A)=0 \quad \text { for all compact } A \subseteq G
$$

is called scattered. If $\mu$ and $\check{\mu}$ are both scattered we say that $\mu$ is symmetrically scattered (we briefly write s.scattered).

It is proved in [DL2] that $\mu$ is scattered if and only if $\lim _{n \rightarrow \infty}\left\|P_{\mu}^{n} f\right\|_{2}=0$ for all $f \in L^{2}(\lambda)$.

Definition 3. A probability measure $\mu$ on $G$ is called concentrated if

(4) there exist a compact set $A \subset G$ and a sequence $g_{n} \in G$ such that $\mu^{* n}\left(g_{n} A\right) \equiv 1$.

If $\mu$ and $\check{\mu}$ are concentrated then $\mu$ is called symmetrically concentrated (briefly, s.concentrated).

For Abelian or countable groups any nonscattered measure is s.concentrated (see [DL1] and [B2]). Moreover, the compact set $A$ in (4) can be taken to be a compact subgroup of $G$ and $g_{n} \in S\left(\mu^{* n}\right)$. We recall (see [C]) that if a measure $\mu$ is not scattered then for every $0<\varepsilon<1$ there exist a compact set $A \subset G$ and a sequence $g_{n} \in G$ such that $\mu^{* n}\left(g_{n} A\right) \geq 1-\varepsilon$ for all $n$. In particular, for a nonscattered probability measure $\mu$ the sequence $\left\{\mu^{* n}\right\}_{n \in \mathbb{N}}$ is shift compact.

Definition 4. A probability measure $\mu$ on a group $G$ is called

- adapted if the closed subgroup $\mathfrak{G}(\mu)$ generated by the support $S(\mu)$ is $G$,

- irreducible if the closed semigroup $\mathfrak{S}(\mu)$ generated $S(\mu)$ is $G$,

- strictly aperiodic if the closed normal subgroup $\mathfrak{H}(\mu)$ a coset of which contains $S(\mu)$ is $G$,

- spread out if $\mu^{* n}$ is not orthogonal to $\lambda$ for some $n$.

It was proved in [DL2] that any adapted, strictly aperiodic, spread out probability measure on $G$ is scattered (actually s.scattered). However, as shown in [B2], there are s.scattered probabilities which are not strictly aperiodic. In this paper we follow some ideas from [B2], so we adopt the following: 
Definition 5. For a probability measure $\mu$ on $G$ the smallest closed subgroup $H \subseteq G$ such that for any $g \in S(\mu)$,

$$
g H=H g \text { and } S(\mu) \subseteq g H,
$$

is denoted by $\mathfrak{h}(\mu)$.

The subgroups $\mathfrak{H}(\mu)$ and $\mathfrak{h}(\mu)$ may be different if $\mu$ is not adapted. However, for adapted $\mu$ they coincide and are described in [DL2] (Proposition 1.1) as the closed subgroup generated by $\bigcup_{n=1}^{\infty}\left(S\left(\mu^{* n} * \check{\mu}^{* n}\right) \cup S\left(\check{\mu}^{* n} *\right.\right.$ $\left.\left.\mu^{* n}\right)\right)$.

3. Residuality of s.scattered measures. In this section we assume that $G$ is a locally compact second countable topological group (therefore a Polish metric space). We study the set $P_{\text {scat }}(G)$ of all scattered probabilities on $G$. We show that a typical scattered (s.scattered) probability measure is not spread out. This means that the class of s.scattered probabilities described by Derriennic and Lin (in [DL2], Theorem 3.5), however large (variation norm dense in $P(G)$ ), is rather small from the category point of view (meager in the weak measure topology).

We begin with the remark that $P_{\text {scat }}(G)$ is a $G_{\delta}$ subset of $P(G)$ for the weak measure topology. First notice that we have the following representation:

$$
P_{\text {scat }}(G)=\bigcap_{j=1}^{\infty} \bigcap_{m=1}^{\infty} \bigcap_{N=1}^{\infty} \bigcup_{n \geq N}\left\{\mu \in P(G):\left\|P_{\mu}^{n} f_{j}\right\|_{2}<1 / m\right\}
$$

where $\left\{f_{j}\right\}_{j=1}^{\infty}$ is a $\|\cdot\|_{2}$ dense subset of $L^{2}(\lambda)$. By the relations mentioned above between the topologies on $P(G)$ and $\mathcal{M}_{2}(G)$, the set $P_{\text {scat }}(G)$ is a weak $G_{\delta}$ subset of $P(G)$. Since the mapping $P(G) \ni \mu \rightarrow \check{\mu} \in P(G)$ is a homeomorphism in the weak measure topology, the set $P_{\text {s.scat }}(G)$ of all s.scattered probabilities on $G$ is again a $G_{\delta}$ set as the intersection of two weak $G_{\delta}$ sets.

Now, let $\mu_{0} \in P(G)$ be absolutely continuous with respect to $\lambda$, and $S\left(\mu_{0}\right)=G$. Clearly such a $\mu_{0}$ exists and by [DL2] it is s.scattered as are all convex combinations $(1-\varepsilon) \nu+\varepsilon \mu_{0}$ where $0<\varepsilon \leq 1$ and $\nu \in P(G)$. So for any locally compact second countable group $G$ the set $P_{\text {s.scat }}(G)$ is a norm variation dense and weak $G_{\delta}$ subset of $P(G)$. In particular, it is residual. The following result shows that spread out s.scattered measures for "continuous" groups $G$ are meager in $P(G)$, and also in $P_{\text {s.scat }}(G)$.

Proposition 1. Let $G$ be a nondiscrete locally compact second countable topological group. Then the set of all s.scattered non-spread out probability measures $\mu$ on $G$ contains a weak dense $G_{\delta}$ subset of $P(G)$. 
Pro of. Let $G_{0}$ be a countable dense subgroup of $G$. For any natural $m$ there exists an open set $U_{m}$ such that $\lambda\left(U_{m}\right)<1 / m$ and $G_{0} \subset U_{m}$. We may assume that the sequence $U_{m}$ is decreasing. The convolution operation $*$ is continuous for the weak measure topology, so for any fixed $m, n \in \mathbb{N}$ the set $D_{m, n}=\left\{\mu \in P(G): \mu^{* n}\left(U_{m}\right)>1 / 2\right\}$ is open in the weak measure topology. Since $P\left(G_{0}\right) \subseteq D_{m, n}$ it follows that $\bigcap_{m=1}^{\infty} \bigcap_{n=1}^{\infty} D_{m, n}=D$ is a weak dense $G_{\delta}$ subset of $P(G)$. By the Baire theorem, $D \cap P_{\text {s.scat }}(G)$ is again a weak dense $G_{\delta}$ subset of $P(G)$. To finish the proof we notice that there is no spread out probability measure in $D$.

4. Concentrated measures on groups with invariant metrics. The results of the previous section prompt the study of s.scattered measures which are not spread out. Even though we do not know how things go in the general case, we settle the problem of s.scattered probabilities for groups having invariant metrics. This generalizes some results of [B2] and of [DL1]. We begin this section with the following lemma, which actually comes from [B2] and is valid for all locally compact $\sigma$-compact Hausdorff groups $G$.

LEMMA 1. For any probability measure $\mu$ on $G$ either there exists a probability measure $\varrho$ on $G$ such that $P_{\check{\mu}^{* n} * \mu^{* n}} \rightarrow P_{\varrho}$ in the weak operator topology (w.o.t.) in $\mathcal{M}_{2}(G)$ or $P_{\mu}^{n} \rightarrow 0$ in the strong operator topology in $\mathcal{L}\left(L^{2}(\lambda)\right)$. Moreover, the measure $\varrho$ satisfies $\check{\mu} * \varrho * \mu=\varrho$.

Proof. Assume that $\left\|P_{\mu}^{n} f\right\|_{2} \nrightarrow 0$ for some $f \in L^{2}(\lambda)$. Then $\mu$ is nonscattered. The results of Csiszár [C] imply that for any positive $\varepsilon$ there exists a compact set $A \subseteq G$ and a sequence $g_{n} \in G$ such that $\mu^{* n}\left(g_{n} A\right) \geq 1-\varepsilon$. So for all $n$ we get

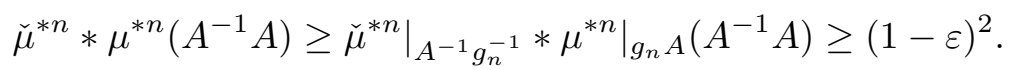

By Prokhorov's theorem the sequence $\check{\mu}^{* n} * \mu^{* n}$ is relatively compact for the weak measure topology (see e.g. Theorem 6.7 in $[\mathrm{P}]$ ). This implies that any limit measure $\varrho$ of the sequence $\check{\mu}^{* n} * \mu^{* n}$ in the vague topology belongs to $P(G)$.

Now let $Q_{1}$ and $Q_{2}$ be arbitrary w.o.t. limit operators of the sequence $P_{\check{\mu}^{* n} * \mu^{* n}}$ on $L^{2}(\lambda)$. We show that they are equal. In fact, for all $f \in L^{2}(\lambda)$ we have

$$
\left\langle Q_{1} f, f\right\rangle=\lim _{n \rightarrow \infty}\left\|P_{\mu}^{n} f\right\|_{2}^{2}=\left\langle Q_{2} f, f\right\rangle,
$$

where $\langle\cdot, \cdot\rangle$ stands for the scalar product in $L^{2}(\lambda)$. So by a standard functional analysis argument $Q_{1}=Q_{2}$. This implies the existence of the w.o.t. limit $\lim _{n \rightarrow \infty} P_{\check{\mu}^{* n} * \mu^{* n}}=Q$. Since the convolution operators form a w.o.t. closed subset of $\mathcal{L}\left(L^{2}(\lambda)\right)$, for some probability measure $\varrho$ on $G$ we have $Q=P_{\varrho}$. Clearly $P_{\check{\mu}} \circ P_{\varrho} \circ P_{\mu}=P_{\varrho}$ so the equality $\check{\mu} * \varrho * \mu=\varrho$ follows. 
Remark 1 . Since the weak and strong operator topologies coincide on $\mathcal{M}_{2}(G)$, the convergence $P_{\check{\mu}^{* n} * \mu^{* n}} \rightarrow P_{\varrho}$ in Lemma 1 is actually in the strong operator topology.

Now we are in a position to formulate the main result of the paper.

THEOREM 1. Let $G$ be a locally compact second countable topological group with an invariant metric d. Then the following conditions are equivalent:

(i) $\mu$ is concentrated,

(ii) $\mu$ is nonscattered,

(iii) there exists $f \in L^{2}(\lambda)$ such that $\lim _{n \rightarrow \infty}\left\|P_{\mu}^{n} f\right\|_{2}>0$,

(iv) there exists $\varrho \in P(G)$ such that $\check{\mu} * \varrho * \mu=\varrho$,

(v) $\lim _{n \rightarrow \infty} L\left(S\left(\mu^{* n}\right), \varepsilon\right)=l_{\varepsilon}<\infty$ for all $\varepsilon>0$,

(vi) the subgroup $\mathfrak{h}(\mu)$ is compact.

Proof. (i) $\Rightarrow$ (ii) is obvious, (ii) $\Rightarrow$ (iii) follows from [DL2], and (iii) $\Rightarrow$ (iv) is a consequence of Lemma 1.

(iv) $\Rightarrow(\mathrm{v})$. For a fixed $\varepsilon>0$ let $\alpha=\sup _{g \in G} \varrho(\bar{K}(g, \varepsilon / 3))$. We may assume that $\bar{K}(g, \varepsilon / 3)$ is compact. By Lemma 3.1 of $[\mathrm{P}]$ the set $\{g \in G: \alpha=$ $\varrho(\bar{K}(g, \varepsilon / 3))\}$ is nonempty and compact. So, for any natural $n$ we get

$$
\alpha=\varrho\left(\bar{K}\left(g_{0}, \varepsilon / 3\right)\right)=\int_{G} \int_{G} \varrho\left(y \bar{K}\left(g_{0}, \varepsilon / 3\right) z^{-1}\right) d \mu^{* n}(y) d \mu^{* n}(z) .
$$

This implies that $\varrho\left(\bar{K}\left(y g_{0} z^{-1}, \varepsilon / 3\right)\right)=\alpha$ for $\mu^{* n} \otimes \mu^{* n}$-almost all $(y, z)$. Now, consider a family $\left\{y_{1} \ldots, y_{l}\right\} \subseteq S\left(\mu^{* n}\right)$ such that $d\left(y_{i}, y_{j}\right) \geq \varepsilon$ if $i \neq j$. For some $z_{0} \in S\left(\mu^{* n}\right)$ we may find $\widetilde{y}_{1}, \ldots, \widetilde{y}_{l} \in S\left(\mu^{* n}\right)$ such that $d\left(y_{j}, \widetilde{y}_{j}\right) \leq$ $\varepsilon / 10, \varrho\left(\bar{K}\left(\widetilde{y}_{j} g_{0} z_{0}^{-1}, \varepsilon / 3\right)\right)=\alpha$, and the $\bar{K}\left(\widetilde{y}_{j} g_{0} z_{0}^{-1}, \varepsilon / 3\right)$ are pairwise disjoint. Clearly,

$$
1 \geq \sum_{j=1}^{l} \varrho\left(\bar{K}\left(\widetilde{y}_{j} g_{0} z_{0}^{-1}, \varepsilon / 3\right)\right)=l \alpha,
$$

so that $l \leq 1 / \alpha$. Finally, for any $\varepsilon>0$ we get

$$
l_{\varepsilon}=\lim _{n \rightarrow \infty} L\left(S\left(\mu^{* n}\right), \varepsilon\right) \leq \frac{1}{\sup _{g \in G} \varrho(\bar{K}(g, \varepsilon / 3))} .
$$

$(\mathrm{v}) \Rightarrow(\mathrm{vi})$. Notice that for any $\varepsilon>0$ the sequence of natural numbers $L\left(S\left(\mu^{* n}\right), \varepsilon\right)$ is nondecreasing. If $l_{\varepsilon}<\infty$ then there exists $N_{\varepsilon}$ such that for all $n \geq N_{\varepsilon}$ it is constant and equal to $l_{\varepsilon}$. The mapping $G \ni g \rightarrow g^{-1} \in G$ is an isometry, so $L\left(S\left(\mu^{* n}\right), \varepsilon\right)=L\left(S\left(\check{\mu}^{* n}\right), \varepsilon\right)$. Applying the triangle inequality we easily get

$$
N\left(S\left(\check{\mu}^{* n} * \mu^{* n}\right), 2 \varepsilon\right) \leq l_{\varepsilon}^{2} \quad \text { and } \quad N\left(S\left(\mu^{* n} * \check{\mu}^{* n}\right), 2 \varepsilon\right) \leq l_{\varepsilon}^{2} .
$$


Hence $N\left(R_{n}, 2 \varepsilon\right) \leq 2 l_{\varepsilon}^{2}$, where $R_{n}=S\left(\check{\mu}^{* n} * \mu^{* n}\right) \cup S\left(\mu^{* n} * \check{\mu}^{* n}\right)$. We notice that $R_{n}$ is a nondecreasing sequence of compact subsets of $G$. Obviously each $R_{n}$ is symmetric $\left(R_{n}^{-1}=R_{n}\right)$ and $e \in R_{n}$. We set $R_{\infty}=\bigcup_{n=1}^{\infty} R_{n}$ and $R=\bar{R}_{\infty}$. The previous estimates imply that $N\left(R_{\infty}, 2 \varepsilon\right) \leq 2 l_{\varepsilon}^{2}$ for any $\varepsilon>0$. So $R_{\infty}$ is relatively compact and thus $R$ is compact. In order to show that $R$ is a subgroup of $G$ it is sufficient to check that it is a semigroup, i.e. $R_{\infty} R_{\infty} \subseteq R_{\infty}$. For this we will examine the products $g_{1} g_{2}$ in the 4 cases: $(\alpha) g_{1}, g_{2} \in S\left(\check{\mu}^{* m} * \mu^{* m}\right),(\beta) g_{1} \in S\left(\check{\mu}^{* m} * \mu^{* m}\right)$ and $g_{2} \in S\left(\mu^{* m} * \check{\mu}^{* m}\right)$, $(\gamma) g_{1} \in S\left(\mu^{* m} * \check{\mu}^{* m}\right)$ and $g_{2} \in S\left(\check{\mu}^{* m} * \mu^{* m}\right),(\delta) g_{1}, g_{2} \in S\left(\mu^{* m} * \check{\mu}^{* m}\right)$ for $m \geq N_{\varepsilon}$.

Since $L\left(S\left(\mu^{* n}\right), \varepsilon\right)=l_{\varepsilon}$ for $n \geq N_{\varepsilon}$ and the mappings $G \ni x \rightarrow$ $g x \in G$ and $G \ni x \rightarrow x g \in G$ are isometries, for any $g \in S\left(\mu^{* k}\right)$ the sets $S\left(\mu^{* n}\right) g$ and $g S\left(\mu^{* n}\right)$ are $\varepsilon$-dense in $S\left(\mu^{*(n+k)}\right)$. This also implies that for all $g \in S\left(\check{\mu}^{* k}\right)=\left(S\left(\mu^{* k}\right)\right)^{-1}$ we have $\operatorname{dist}\left(S\left(\mu^{* n}\right), S\left(\mu^{*(n+k)}\right) g\right) \leq \varepsilon$ and $\operatorname{dist}\left(S\left(\mu^{* n}\right), g S\left(\mu^{*(n+k)}\right)\right) \leq \varepsilon$ for $n \geq N_{\varepsilon}$ and $k \geq 1$. In particular, $\operatorname{dist}\left(S\left(\mu^{* n}\right), S\left(\check{\mu}^{* k}\right) S\left(\mu^{*(n+k)}\right)\right) \leq \varepsilon, \operatorname{dist}\left(S\left(\mu^{* n}\right), S\left(\mu^{*(n+k)}\right) S\left(\check{\mu}^{* k}\right)\right) \leq \varepsilon$, $\operatorname{dist}\left(S\left(\check{\mu}^{* n}\right), S\left(\check{\mu}^{*(n+k)}\right) S\left(\mu^{* k}\right)\right) \leq \varepsilon, \operatorname{dist}\left(S\left(\check{\mu}^{* n}\right), S\left(\mu^{* k}\right) S\left(\check{\mu}^{*(n+k)}\right)\right) \leq \varepsilon$.

Now, consider $(\alpha)$ and take natural numbers $n, k$ such that $k, n-k \geq m$. Then

$$
\begin{aligned}
g_{1} g_{2} & \in S\left(\check{\mu}^{* n} * \mu^{* n}\right) S\left(\check{\mu}^{* k} * \mu^{* k}\right) \subseteq S\left(\check{\mu}^{* n}\right) \overline{\mathbf{K}}\left(S\left(\mu^{*(n-k)}\right), \varepsilon\right) S\left(\mu^{* k}\right) \\
& \subseteq S\left(\check{\mu}^{* n}\right) \overline{\mathbf{K}}\left(S\left(\mu^{*(n-k)} * \mu^{* k}\right), \varepsilon\right) \subseteq \overline{\mathbf{K}}\left(S\left(\check{\mu}^{* n} * \mu^{* n}\right), \varepsilon\right) .
\end{aligned}
$$

In particular, $d\left(g_{1} g_{2}, R\right) \leq 2 \varepsilon$ for any $\varepsilon>0$. Since $R$ is closed, $g_{1} g_{2} \in R$.

In the case $(\beta)$ take $n, k \geq m$. Then

$$
\begin{aligned}
g_{1} g_{2} & \in S\left(\check{\mu}^{* n} * \mu^{* n}\right) S\left(\mu^{* k} * \check{\mu}^{* k}\right)=S\left(\check{\mu}^{* n}\right) S\left(\mu^{*(n+k)}\right) S\left(\check{\mu}^{* k}\right) \\
& \subseteq \overline{\mathbf{K}}\left(S\left(\mu^{* k}\right), 2 \varepsilon\right) S\left(\check{\mu}^{* k}\right) \subseteq \overline{\mathbf{K}}\left(S\left(\mu^{* k} * \check{\mu}^{* k}\right), 2 \varepsilon\right) \text { for any } \varepsilon>0,
\end{aligned}
$$

hence again $g_{1} g_{2} \in R$. Since the cases $(\delta)$ and $(\gamma)$ are symmetric respectively to $(\alpha)$ or $(\beta)$, we thus proved that $R$ is a compact subgroup of $G$.

Obviously, $S(\check{\mu} * \mu) \subseteq R$, so $S(\mu) \subseteq g R$ for any $g \in S(\mu)$. To show that $R$ is a normal subgroup of $\mathfrak{G}(\mu)$ consider $\left\{g \in G: g R g^{-1}=R\right\}$. Clearly this is a subgroup of $G$. For any $g \in S(\mu), \varepsilon>0$ and $n$ large enough we have

$$
\begin{aligned}
g S\left(\check{\mu}^{* n} * \mu^{* n}\right) g^{-1} & \subseteq \overline{\mathbf{K}}\left(S\left(\check{\mu}^{*(n-1)}\right), \varepsilon\right) \overline{\mathbf{K}}\left(S\left(\mu^{*(n-1)}\right), \varepsilon\right) \\
& \subseteq \overline{\mathbf{K}}\left(S\left(\check{\mu}^{*(n-1)} * \mu^{*(n-1)}\right), 2 \varepsilon\right) \subseteq \overline{\mathbf{K}}(R, 2 \varepsilon)
\end{aligned}
$$

and

$$
g S\left(\mu^{* n} * \check{\mu}^{* n}\right) g^{-1} \subseteq R .
$$

This implies that $g R_{\infty} g^{-1} \subseteq \overline{\mathbf{K}}(R, 4 \varepsilon)$ for any $\varepsilon>0$. By the continuity of group operations we get $g R g^{-1} \subseteq R$ for all $g \in S(\mu)$. Since $R$ is compact and $R \ni x \rightarrow g x g^{-1} \in R$ is an isometry, by the classical topological argument we get $g R g^{-1}=R$ if $g \in S(\mu)$. This yields $g R g^{-1}=R$ for all $g \in \mathfrak{G}(\mu)$ and 
so $R$ is a normal subgroup of $\mathfrak{G}(\mu)$. Hence $\mathfrak{h}(\mu) \subseteq R$. Since $S\left(\check{\mu}^{* n} * \mu^{* n}\right) \cup$ $S\left(\mu^{* n} * \check{\mu}^{* n}\right) \subseteq \mathfrak{h}(\mu)$ for any $n$, we have $R \subseteq \mathfrak{h}(\mu)$, and finally $\mathfrak{h}(\mu)=R$ is a compact subgroup of $G$.

(vi) $\Rightarrow(\mathrm{i})$. Consider the compact set $A=\mathfrak{h}(\mu)$. For any $g \in S(\mu)$ we have

$$
S\left(\mu^{* n}\right) \subseteq g \mathfrak{h}(\mu) \ldots g \mathfrak{h}(\mu)=g^{n} \mathfrak{h}(\mu),
$$

so $\mu^{* n}\left(g^{n} A\right) \equiv 1$ for all $n$. It follows that $\mu$ is concentrated.

Problem. For which locally compact $\sigma$-compact Hausdorff topological groups does the characterization obtained in Theorem 1 hold?

5. S.concentrated and spread out measures. For the rest of our paper we drop the assumption that the group $G$ has an invariant metric or even that it is metrizable. But we still assume it is locally compact, $\sigma$-compact and Hausdorff.

For a fixed probability $\mu$ on a unimodular group $G$ we define the linear operator

$$
T_{\mu} f(x)=\int_{G} \int_{G} f\left(y x z^{-1}\right) d \mu(y) d \mu(z) .
$$

Clearly, $T_{\mu}$ is a linear positive contraction on $L^{p}(\lambda), 1 \leq p \leq \infty$, and $C_{0}(G)$. Moreover, on $L^{1}(\lambda)$ it is stochastic (i.e. for any nonnegative $f \in L^{1}(\lambda)$ we have $T_{\mu} f \geq 0$ and $\left\|T_{\mu} f\right\|_{1}=\|f\|_{1}$ ). Notice that for any $1 \leq p<\infty$ the adjoint operator to $T_{\mu}$ is $T_{\tilde{\mu}}$. On $L^{1}(\lambda), T_{\mu}$ is the restriction of the stochastic linear operator $\nu \rightarrow \breve{\mu} * \nu * \mu$ acting on the (AL) Banach lattice $M(G)$.

Proposition 2. Let $G$ be a locally compact $\sigma$-compact Hausdorff topological group. If there exists an adapted spread out measure $\mu \in P(G)$ such that both $\mu$ and $\check{\mu}$ are nonscattered then $G$ is unimodular.

Proof. Assume that $\mu$ is not scattered. Then by Lemma 1 there exists $\varrho \in P(G)$ which satisfies $\check{\mu} * \varrho * \mu=\varrho$. Since $L^{1}(\lambda)$ is a two-sided ideal in the convolution Banach algebra $M(G)$, any such $\varrho$ belongs to $L^{1}(\lambda)$. In fact, first we notice that for a spread out measure $\mu \in P(G)$ we have $\left\|\mu_{\mathrm{ac}}^{* n}\right\|=$ $\left\|\check{\mu}_{\mathrm{ac}}^{* n}\right\| \nearrow 1$, where $\nu_{\mathrm{ac}}$ stands for the absolutely continuous component of $\nu$. This implies that $\left\|\varrho_{\mathrm{ac}}\right\| \geq\left\|\check{\mu}^{* n} * \varrho * \mu_{\mathrm{ac}}^{* n}\right\|=\left\|\mu_{\mathrm{ac}}^{* n}\right\| \nearrow 1$. In particular, $\left\|\varrho_{\text {ac }}\right\|=1$, so $\varrho=\varrho_{\text {ac }} \in L^{1}(\lambda)$. For $\lambda$-almost all $x \in G$ we have

$$
\frac{d \varrho}{d \lambda}(x)=\int_{G} \int_{G} \Delta(y) \frac{d \varrho}{d \lambda}\left(y x z^{-1}\right) d \mu(y) d \mu(z),
$$

where $\Delta$ stands for the modular function on $G$. From the estimation

$$
\sup _{r \in \mathbb{R}_{+}}\left(\mu \circ \Delta^{-1}\right)^{* n}(r \Delta(F)) \geq \sup _{g \in G} \mu^{* n}(g F)
$$


we conclude that the probability measure $\mu \circ \Delta^{-1}$ on the multiplicative group $\mathbb{R}_{+}$is nonscattered. By [DL1] it must be a Dirac delta $\delta_{a}$. Let us modify (7) and define the positive linear contractions on $L^{p}(\lambda)$ by

$$
T_{\mu, p} f(x)=\int_{G} \int_{G}(\Delta(y))^{1 / p} f\left(y x z^{-1}\right) d \mu(y) d \mu(z) .
$$

For unimodular $G$ the operators $T_{\mu, p}$ and $T_{\mu}$ are the same. But if $G$ is not unimodular and $\mu$ is adapted then $\Delta(S(\mu))=a \neq 1$.

So, $T_{\mu, p} f(x)=a^{1 / p} \int_{G} \int_{G} f\left(y x z^{-1}\right) d \mu(y) d \mu(z)$ for any $1 \leq p<\infty$ and $f \in L^{p}(\lambda)$. Let $F \subseteq G$ be compact such that $\int_{F}(d \varrho / d \lambda) d \lambda=\varrho(F) \geq 1-\varepsilon$ and $0 \leq f \leq d \varrho / d \lambda$ be a function from $L^{2}(\lambda)$ such that $\|d \varrho / d \lambda-f\|_{1} \leq \varepsilon$ for some $0<\varepsilon<1 / 2$. Then

$$
\begin{aligned}
\int_{F} T_{\mu, 1}^{n} f d \lambda & =\int_{F} T_{\mu, 1}^{n}\left(f-\frac{d \varrho}{d \lambda}\right) d \lambda+\int_{F} T_{\mu, 1}^{n}\left(\frac{d \varrho}{d \lambda}\right) d \lambda \\
& \geq 1-\varepsilon-\left\|f-\frac{d \varrho}{d \lambda}\right\|_{1} \geq 1-2 \varepsilon .
\end{aligned}
$$

On the other hand, the Cesàro means satisfy

$$
\begin{aligned}
\frac{1}{N} \sum_{n=0}^{N-1} \int_{F} & T_{\mu, 1}^{n} f d \lambda \\
= & \frac{1}{N} \sum_{n=0}^{N-1} a^{n / 2} \int_{F} \int_{G} \int_{G} a^{n / 2} f\left(y x z^{-1}\right) d \mu^{* n}(y) d \mu^{* n}(z) d \lambda(x) \\
= & \frac{1}{N} \sum_{n=0}^{N-1} a^{n / 2} \int_{F} T_{\mu, 2}^{n} f d \lambda \leq \frac{1}{N} \sum_{n=0}^{N-1} a^{n / 2}\|f\|_{2}\left\|\mathbf{1}_{F}\right\|_{2} \\
= & \frac{\|f\|_{2}\left\|\mathbf{1}_{F}\right\|_{2}}{N} \cdot \frac{1-a^{N / 2}}{1-a^{1 / 2}} \rightarrow 0 \quad \text { as } N \rightarrow \infty
\end{aligned}
$$

if $0<a<1$. For $a>1$ we can consider a nonscattered spread out measure $\check{\mu}$ for which $\Delta(S(\check{\mu}))=1 / a<1$. So, $\Delta(S(\mu)) \equiv 1$. If $\mu$ is adapted then $\Delta$ is 1 on the whole group, and $G$ is unimodular.

Remark 2. It is not hard to notice that if $\mu$ is not adapted then in the above proposition we only obtain the unimodularity of the subgroup $\left(\mathfrak{G}(\mu),\left.\lambda\right|_{\mathfrak{G}(\mu)}\right)$.

COROLlary 1. Let $\mu$ be a spread out probability measure on a locally compact $\sigma$-compact Hausdorff topological group. If $\mathfrak{G}(\mu)$ is not unimodular then either $\mu$ or $\check{\mu}$ is scattered. 
The next result is a version of Theorem 1 for spread out measures. Instead of groups with invariant metrics we obtain the corresponding equivalences for the whole class of unimodular groups.

TheOREM 2. Let $G$ be a unimodular locally compact $\sigma$-compact Hausdorff topological group. For any spread out probability measure $\mu$ on $G$ the following are equivalent:

(I) $\mu$ is concentrated,

(II) $\mu$ is nonscattered,

(III) there exists $f \in L^{2}(\lambda)$ such that $\lim _{n \rightarrow \infty}\left\|P_{\mu}^{n} f\right\|_{2}>0$,

(IV) there exists $\varrho \in P(G)$ such that $\check{\mu} * \varrho * \mu=\varrho$ and $d \varrho / d \lambda=\Theta \in$ $L^{1}(\lambda) \cap L^{\infty}(\lambda) \subseteq L^{2}(\lambda)$,

(VI) the subgroup $\mathfrak{h}(\mu)$ is compact and has nonempty interior.

Proof. $(\mathrm{I}) \Rightarrow(\mathrm{II}) \Rightarrow(\mathrm{III})$ and $(\mathrm{VI}) \Rightarrow(\mathrm{I})$ can be proved in the same way as in the proof of Theorem 1 .

$(\mathrm{III}) \Rightarrow(\mathrm{IV})$. By Lemma 1 and Proposition 2 there exists $\varrho \in P(G)$ which satisfies $\check{\mu} * \varrho * \mu=\varrho$ and is absolutely continuous with respect to $\lambda$. By the von Neumann mean ergodic theorem (see e.g. [CFS], Theorem 4, p. 34), for any $f \in L^{2}(\lambda)$ the Cesàro means $(1 / N) \sum_{n=0}^{N-1} T_{\mu}^{n} f$ converge in the $L^{2}$ norm to some $T_{\mu}$-invariant function $f_{*} \in L^{2}(\lambda)$. We notice that $T_{\mu}$ is a positive linear contraction on $L^{\infty}(\lambda)$ as well, so $\left\|f_{*}\right\|_{\infty} \leq\|f\|_{\infty}$. Now choose $f \in L^{1}(\lambda) \cap L^{\infty}(\lambda) \subseteq L^{2}(\lambda)$ such that $0 \leq f \leq d \varrho / d \lambda$ and $\|f-d \varrho / d \lambda\|<1 / 2$. Since $d \varrho / d \lambda$ is $T_{\mu}$-invariant, $\int_{F} T_{\mu}^{n} f d \lambda>1 / 2$ for some compact set $F$ and all $n$. We therefore conclude that the limit function $\Theta=f_{*} \in L^{2}(\lambda)$ is $T_{\mu}$-invariant, positive and nontrivial.

$$
\begin{aligned}
& (\mathrm{IV}) \Rightarrow(\mathrm{VI}) \text {. We notice that } \\
& \Theta(\cdot)=\int_{G} \int_{G} \Theta\left(y \cdot z^{-1}\right) d \mu^{* n}(y) d \mu^{* n}(z)=\int_{G} \int_{G} \Theta_{y} \Theta_{z^{-1}} d \mu^{* n}(y) d \mu^{* n}(z)
\end{aligned}
$$

for all $n$. By uniform convexity of $L^{2}(\lambda)$ (only strict convexity is exploited) and the fact that $L^{2}(\lambda) \ni f \rightarrow{ }_{y} f_{z^{-1}} \in L^{2}(\lambda)$ is a linear isometry for any fixed $y, z \in G$, we get

$$
{ }_{y} \Theta_{z^{-1}}=\Theta, \quad{ }_{y} \Theta=\Theta_{z}, \quad{ }_{y^{-1}} \Theta=\Theta_{z^{-1}} \quad \text { for } y, z \in S\left(\mu^{* n}\right) .
$$

Since we also have

$$
\Theta(\cdot)=T^{* n} \Theta(\cdot)=\int_{G} \int_{G} y \Theta_{z^{-1}}(\cdot) d \check{\mu}^{* n}(y) d \check{\mu}^{* n}(z),
$$

it follows that

$$
{ }_{y} \Theta_{z^{-1}}=\Theta, \quad{ }_{y} \Theta=\Theta_{z}, \quad{ }_{y^{-1}} \Theta=\Theta_{z^{-1}} \quad \text { for } y, z \in S\left(\check{\mu}^{* n}\right) .
$$


Combining (9) and $\left(9^{*}\right)$ we can easily obtain $\Theta_{y^{-1} z}=\Theta=\Theta_{y z^{-1}}$ for all $y, z$ in $S\left(\mu^{* n}\right)$ or $S\left(\check{\mu}^{* n}\right)$. Now the inclusion

$$
\overline{\bigcup_{n=1}^{\infty} S\left(\check{\mu}^{* n} * \mu^{* n}\right) \cup S\left(\mu^{* n} * \check{\mu}^{* n}\right)} \subseteq \mathcal{G}_{\Theta}:=\left\{t \in G: \Theta_{t}=\Theta\right\}
$$

is obvious. Clearly $\mathcal{G}_{\Theta}$ is a closed subgroup of $G$. Moreover, since $\Theta \in L^{2}(\lambda)$, it is compact. The inclusion (10) yields that $\mathcal{G}_{\Theta}$ has nonempty interior, so has strictly positive and finite Haar measure. For any $g \in S(\mu)$ we have $S(\mu) \subseteq g \mathcal{G}_{\Theta}$. Let $g \in S(\mu)$ and $t \in \mathcal{G}_{\Theta}$ be arbitrary. Then by (9),

$$
\Theta\left(\cdot g t g^{-1}\right)=\Theta_{g^{-1}}(\cdot g t)={ }_{g^{-1}} \Theta_{t}(\cdot g)={ }_{g^{-1}} \Theta(\cdot g)=\Theta_{g^{-1}}(\cdot g)=\Theta(\cdot),
$$

so $g g^{-1} \in \mathcal{G}_{\Theta}$. This means that $g \mathcal{G}_{\Theta} g^{-1} \subseteq \mathcal{G}_{\Theta}$ for all $g \in S(\mu)$. By unimodularity $\lambda\left(g \mathcal{G}_{\Theta} g^{-1}\right)=\lambda\left(\mathcal{G}_{\Theta}\right)$, so $g \mathcal{G}_{\Theta} g^{-1}$ is dense in $\mathcal{G}_{\Theta}$. Since $\mathcal{G}_{\Theta}$ is compact, $g \mathcal{G}_{\Theta} g^{-1}=\mathcal{G}_{\Theta}$ for all $g \in S(\mu)$. The last equality easily extends to all $g \in \mathfrak{G}(\mu)$. Finally, $g \mathcal{G}_{\Theta}=\mathcal{G}_{\Theta} g$ and $S(\mu) \subseteq g \mathcal{G}_{\Theta}$ for all $g \in S(\mu)$. This implies that $\mathfrak{h}(\mu) \subseteq \mathcal{G}_{\Theta}$, so $\mathfrak{h}(\mu)$ is compact. By the spread out assumption it has nonempty interior as well.

COROLlaRY 2. Let $G$ be a noncompact topological group with the properties of Theorem 2. If $\mu \in P(G)$ is spread out then it is scattered if and only if it is s.scattered and only if $\mathfrak{h}(\mu)$ is noncompact.

The above result corresponds to Theorem 3.5 of [DL2], where it is shown that a spread out adapted measure $\mu \in P(G)$ is scattered if $G / \mathfrak{h}(\mu) \neq$ $\mathbb{Z}$. For this we show that for an adapted, spread out and nonscattered $\mu$ the condition $G / \mathfrak{h}(\mu) \neq \mathbb{Z}$ implies unimodularity of $G$. In fact, if $\mu$ (or $\check{\mu}$ ) is nonscattered then by (8) the measure $\mu \circ \Delta^{-1}$ is nonscattered on $\mathbb{R}_{+}$. Applying Theorem 4 of [DL1] we conclude that it is the Dirac delta $\delta_{a}$ for some $a \in \mathbb{R}_{+}$. Therefore $\Delta(S(\mu)) \equiv a$, which gives

$$
\mathfrak{h}(\mu)=\overline{\mathcal{G}\left(\bigcup_{n=1}^{\infty}\left(S\left(\check{\mu}^{* n} * \mu^{* n}\right) \cup S\left(\mu^{* n} * \check{\mu}^{* n}\right)\right)\right)} \subseteq G_{\Delta} .
$$

By Theorem 2.9 of $[\mathrm{DL} 2], \operatorname{card}\left(G / G_{\Delta}\right) \leq \operatorname{card}(G / \mathfrak{h}(\mu))<\infty$. This implies that $\Delta(G)$ is a finite subgroup of $\mathbb{R}_{+}$, so it must be trivial, i.e. $G$ is unimodular, and we apply our Theorem 2 .

Now let us present another related result.

COROllary 3. Let $G$ be a locally compact $\sigma$-compact Hausdorff topological group. If there exists an adapted, spread out, nonscattered measure $\mu \in P(G)$ such that $G / \mathfrak{h}(\mu) \neq \mathbb{Z}$ then $G$ must be compact.

Proof. By the above considerations, $G$ is unimodular. So, Theorem 2 shows that $\mathfrak{h}(\mu)$ is compact, which easily gives the compactness of $G$. 
We end the paper with a characterization of concentrated measures on connected groups:

COROLlary 4. Let $G$ be a locally compact, $\sigma$-compact, noncompact, connected Hausdorff topological group. For any adapted spread out measure $\mu \in P(G)$ we have

(a) $\mu$ is s.scattered if and only if $\mathfrak{h}(\mu)$ is not compact,

(b) $\mu$ is s.concentrated if and only if $\mathfrak{h}(\mu)$ is compact.

Pro of. For unimodular groups, (a) and (b) can easily be inferred from Theorem 2. By Theorem 2.9 of [DL2] and previous arguments, if there exists an adapted, spread out, nonscattered measure $\mu \in P(G)$ then either $G$ is compact or $G / \mathfrak{h}(\mu)=\mathbb{Z}$. Let us discuss the latter alternative. Since for a nonscattered spread out measure $\mu$ (or $\check{\mu}$ ) we have $\mathfrak{h}(\mu) \subseteq G_{\Delta}$, for nonunimodular connected groups $G$ we would have

$$
\aleph_{0}=\operatorname{card}(G / \mathfrak{h}(\mu)) \geq \operatorname{card}\left(G / G_{\Delta}\right)=\operatorname{card}\left(\mathbb{R}_{+}\right) .
$$

Therefore, if $G / \mathfrak{h}(\mu)=\mathbb{Z}$, then $\mu$ is s.scattered and $\mathfrak{h}(\mu)$ is not compact.

\section{References}

[B1] W. Bartoszek, On the asymptotic behaviour of iterates of positive linear operators, Notices South African Math. Soc. 25 (1993), 48-78.

[B2] -, On concentration functions on discrete groups, Ann. Probab., to appear.

[B3] -, On the equation $\check{\mu} * \varrho * \mu=\varrho$, Demonstratio Math., to appear.

[C] I. Csiszár, On infinite products of random elements and infinite convolutions of probability distributions on locally compact groups, Z. Wahrsch. Verw. Gebiete 5 (1966), 279-299.

[CFS] I. P. Cornfeld, S. V. Fomin and Ya. G. Sinai, Ergodic Theory, Springer, New York, 1981

[DL1] Y. Derriennic et M. Lin, Sur le comportement asymptotique de puissances de convolution d'une probabilité, Ann. Inst. H. Poincaré 20 (1984), 127-132.

[DL2] -, - Convergence of iterates of averages of certain operator representations and of convolution powers, J. Funct. Anal. 85 (1989), 86-102.

[H] H. Heyer, Probability Measures on Locally Compact Groups, Springer, Berlin, 1977.

[HR] E. Hewitt and K. A. Ross, Abstract Harmonic Analysis I, Springer, Berlin, 1963.

[HM] K. H. Hofmann and A. Mukherjea, Concentration functions and a class of non-compact groups, Math. Ann. 256 (1981), 535-548.

[M] A. Mukherjea, Limit theorems for probability measures on non-compact groups and semigroups, Z. Wahrsch. Verw. Gebiete 33 (1976), 273-284.

[P] K. R. Parthasarathy, Probability Measures on Metric Spaces, Academic Press, New York, 1967. 
[T] A. Tortrat, Lois de probabilité sur un espace topologique complétement régulier et produits infinis à termes indépendants dans un groupe topologique, Ann. Inst. H. Poincaré Sect. B 1 (1965), 217-237

DEPARTMENT OF MATHEMATICS, APPLIED MATHEMATICS AND ASTRONOMY UNIVERSITY OF SOUTH AFRICA

P.O. BOX 392

0001 PRETORIA, SOUTH AFRICA

E-mail: BARTOWK@RISC5.UNISA.AC.ZA

Reçu par la Rédaction le 6.5.1993

Révisé le 14.10.1993 